\title{
Site-1 protease cleavage site is important for the ER stress-induced activation of membrane-associated transcription factor bZIP28 in Arabidopsis
}

\author{
SUN Le, ZHANG Shuang-Shuang, LU Sun-Jie \& LIU Jian-Xiang * \\ State Key Laboratory of Genetic Engineering, Institute of Plant Biology, School of Life Sciences, Fudan University, Shanghai 200433, China
}

Received August 11, 2014; accepted December 26, 2014; published online January 27, 2015

\begin{abstract}
Many sources of stress cause accumulation of unfolded or misfolded proteins in endoplasmic reticulum (ER), which elicits the unfolded protein response (UPR) to either promote cell survival or programmed cell death depending on different developmental context or stress severity. The Arabidopsis membrane-associated transcription factor, bZIP28, is the functional equivalent of mammalian ATF6, which relocates from the ER to the Golgi where it is proteolytically processed and released from the membrane to the nucleus to mediate the UPR. Although the canonical site-1 protease (S1P) cleavage site on the ER lumen-facing domain is well conserved between bZIP28 and ATF6, the importance of S1P cleavage on bZIP28 has not been experimentally demonstrated. Here we provide genetic evidence that the RRIL ${ }^{573}$ site, but not the RVLM ${ }^{373}$ site, on the lumen-facing domain of bZIP28 is critical for the biological function of bZIP28 under ER stress condition. Further biochemistry and cell biology studies demonstrated that the RRIL ${ }^{573}$ site, but not the RVLM ${ }^{373}$ site, is required for proteolytic processing and nuclear relocation of bZIP28 in response to ER stress. Our results reveal that S1P cleavage site plays a pivotal role in activation and function of bZIP28 during UPR in plants.
\end{abstract}

membrane-associated transcription factor, ER stress, unfolded protein response, bZIP28, S1P, S2P

Citation: Sun L, Zhang SS, Lu SJ, Liu JX. Site-1 protease cleavage site is important for the ER stress-induced activation of membrane-associated transcription factor bZIP28 in Arabidopsis. Sci China Life Sci, 2015, 58: 270-275, doi: 10.1007/s11427-015-4807-6

In eukaryotic cells, all the secreted proteins and most of the membrane proteins including membrane receptors enter the endoplasmic reticulum (ER) for protein folding and post-translational modifications [1]. When unfolded or misfolded proteins accumulate in ER, a well-conserved response called the unfolded protein response (UPR) operates to mitigate the ER stress by coordinating the protein folding demand with protein folding capacity [2-5]. UPR is fundamentally important not only for development but also for environmental stress adaptation [3,6,7].

In mammals, the UPR encompasses three pathways, the IRE1, PERK and ATF6 pathways [1]. The IRE1 pathway is

*Corresponding author (email: jianxiangliu@ fudan.edu.cn) the most conserved branch among yeast, plants and human. The ER-localized type I membrane protein IRE1 (for inositol requiring kinase 1) has an $\mathrm{N}$-terminal ER stress sensing domain in the ER-lumen and C-terminal protein kinase and RNase domain facing the cytosol [8,9]. Oligomerization of IRE1 under ER stress conditions activates its cytosolic kinase and endo-ribonuclease activity [10], leading to non-conventional splicing of "intron" sequences presented on the mRNAs of yeast $H A C l$, mammalian $X B P I$ or plant bZIP60 [11-18]. Mammalian cells also have the PERK (for dsRNA-activated protein kinase-like ER kinase) pathway, which has not been found in yeast and plants [19]. ER stress induces activation of the ER-localized PERK to attenuate bulk protein translation by phosphorylating the eukaryotic 
translation initiation factor 2A subunit (eIF2A) [20].

ATF6 (for activating transcription factor 6) is a type II membrane-associated bZIP transcription factor. In response to ER stress, ATF6 relocates from the ER to the Golgi where it is processed by two Golgi-resident site-1 protease (S1P) and site-2 protease (S2P) [21]. Although ortholog of ATF6 is not presented in the Arabidopsis genome, the ER membrane-associated bZIP transcription factor bZIP28 is found to be the functional equivalent of mammalian ATF6, which is activated in a similar way to ATF6 [22-24]. The type I membrane protein site-1 protease (S1P), also known as the subtilisin kexin isozyme-1 (SKI-1), is a subtilisin-like serine protease (subtilase) that is conserved between mammals and plants $[25,26]$. Cleavage of ATF6 by S1P reduces the size of the lumen-facing domain of ATF6, which enables more efficient sequential cleavage of ATF6 within the Golgi membrane by site- 2 protease (S2P) [27]. Although bZIP28 has little sequence similarity to ATF6, the canonical S1P cleavage site is well conserved between bZIP28 and ATF6 [22]. However, the importance of the S1P cleavage site in the lumen-facing domain of bZIP28 has not been experimentally demonstrated.

In the current study, we have examined function of the S1P cleavage site presented in bZIP28 in Arabidopsis. Genetic complementation analysis showed that the canonical S1P site RRIL ${ }^{573}$ of bZIP28 is critical for its biological function while the RVLM ${ }^{373}$ site of bZIP28 is less important. Subsequently protein immuno-blotting and sub-cellular localization studies provided evidence that S1P cleavage at RRIL $^{573}$ site is required for proteolytic processing and nuclear relocation of bZIP28 under ER stress condition in Arabidopsis.

\section{Materials and methods}

All plant materials were grown under standard conditions as reported previously [28]. For phenotypic analysis of ER stress sensitivity, seeds were germinated on agar plates containing half-strength Murashige and Skoog (MS) salts, $1.2 \%$ sucrose, $0.05 \% \mathrm{MES}$, plus or minus tunicamycin (TM, $0.05 \mu \mathrm{g} \mathrm{mL}^{-1}$ ), $\mathrm{pH}$ 5.7. Seedlings were photographed and root length was measured 10 days after germination. For gene expression analysis and Western blotting, 10-day-old seedlings grown on 1/2 MS plates were transferred to liquid $1 / 2 \mathrm{MS}$ medium plus or minus TM $\left(5 \mu \mathrm{g} \mathrm{mL}^{-1}\right)$ or DTT (2 $\mathrm{mmol} \mathrm{L}^{-1}$ ) for $4 \mathrm{~h}$, and whole seedlings were harvested for RNA or total protein extraction, respectively. First-strand cDNA was synthesized using Moloney Murine Leukemia Virus (M-MLV, Invitrogen, USA) reverse transcriptase according to the manufacturer's instructions. Primers for RT-PCR were the same as previously reported [28]. For complementation analysis, the full-length of native or mutated form of bZIP28 was expressed driven by the bZIP28 native promoter [28]. For protein sub-cellular localization and processing, N-terminus of bZIP28 (aa1-300) was replaced by GFP tag and 4× MYC tag and over-expressed using the $35 \mathrm{~S}$ promoter [28]. The mutated forms of bZIP28 were generated using a Quick-Change Site-directed Mutagenesis Kit (Stratagene, USA). For stable transformation, various constructs were introduced into the wild-type (wt) or the double mutant (zip28zip60) of bZIP28 and bZIP60 by floral dip [29]. For confocal analysis, various constructs were co-transformed with the ER marker [30], Golgi marker [30] or the nucleus marker [31] in tobacco (Nicotiana benthamiana). After agro-infiltration for 2-3 d, tobacco epidermal leaves were treated with or without $2 \mathrm{mmol} \mathrm{L}^{-1}$ DTT for $4 \mathrm{~h}$ and observed under confocal microscope (Zeiss LSM A710). All the phenotypic data in the paper were subjected to Student's $t$-test.

\section{Results and discussion}

\subsection{The S1P cleavage site RRIL ${ }^{573}$ on the lumen-facing domain of bZIP28 is important for its biological function}

$\mathrm{S} 1 \mathrm{P}$ translates as a preprodomain-containing protein with a transmembrane domain near its C-terminus, which undergoes autocatalytic maturation in the ER through proteolytic cleavages $[25,26]$. Other substrates of S1P include the sterol-regulatory element binding protein (SREBP), ATF6, Luman/CREB3 and old astrocyte specifically induced substance (OASIS) [21,32-34]. Extensive biochemical studies conclude that S1P processes precursors on the consensus motif $(\mathrm{R} / \mathrm{K}) \mathrm{X}$ (hydrophobic) $\mathrm{Z}$ where $\mathrm{Z}$ is any amino acid, preferentially Leu or Thr, but excluding Val, Pro, Glu, Asp, or Cys [25,35]. We compared the putative S1P cleavage sites of Arabidopsis bZIP28 and its paralogs bZIP17 and bZIP49, as well as the Arabidopsis S1P maturation sites with that of human SREBP, ATF6 and S1P prosegments. It was found that Arg at P4 site and I/L at P2 site are well conserved (Table 1). In order to know whether site A and site B of bZIP28 are indeed processing sites, genetic complementation analysis was performed. Previous studies have successfully demonstrated the important roles of bZIP28 and bZIP60 in plant UPR [22,31,36,37]. Knock-outs both the $b Z I P 28$ and $b Z I P 60$ confer high ER stress sensitivity, and restoring the expression of either $b Z I P 28$ or $b Z I P 60$ with the respective native promoter rescues the ER stress sensitive phenotype of the double mutant zip28zip60 [28]. Mutated forms of bZIP28 in which RVLM ${ }^{373}$ and RRIL ${ }^{573}$ were replaced with $\mathrm{GVSM}^{373}$ and GGIL ${ }^{573}$, respectively (Figure 1A), were expressed in the zip28zip60 mutant plants under the bZIP28 native promoter. In each genetic complementation experiment, at least five transgenic lines were examined. It was found that the GGIL ${ }^{573}$ and $\mathrm{GVSM}^{373}$ expressing plants grew as well as the wild-type (wt) plants and the zip28zip60 $(\mathrm{dm})$ mutant plants under normal growth condition (Figure 1B and C). When the ER stress inducer tunicamycin (TM) was added into the growth medium, the 
Table 1 S1P cleavage sites in human and Arabidopsis ${ }^{\text {a) }}$

\begin{tabular}{|c|c|c|c|c|c|c|c|c|c|}
\hline \multirow{2}{*}{ Precursor protein } & \multicolumn{9}{|c|}{ S1P cleavage site sequence } \\
\hline & $\mathrm{P} 6$ & $\mathrm{P} 5$ & $\mathrm{P} 4$ & $\mathrm{P} 3$ & $\mathrm{P} 2$ & P1 & $\mathrm{P} 1^{\prime}$ & $\mathrm{P} 2^{\prime}$ & $\mathrm{P}^{\prime}$ \\
\hline Human pro-S1P Site B & $\mathrm{V}$ & $\mathrm{F}$ & $\mathbf{R}$ & $\mathrm{S}$ & $\mathbf{L}$ & $\mathrm{K} \downarrow$ & $\mathbf{Y}$ & $\mathrm{A}$ & $\mathrm{E}$ \\
\hline Human pro-S1P Site B' & $\mathrm{P}$ & $\mathrm{Q}$ & $\mathbf{R}$ & $\mathrm{K}$ & $\mathbf{V}$ & $\mathrm{F} \downarrow$ & $\mathrm{R}$ & $\mathrm{S}$ & $\mathrm{L}$ \\
\hline Human pro-S1P Site C & $\mathrm{S}$ & $\mathrm{S}$ & $\mathbf{R}$ & $\mathrm{R}$ & $\mathbf{L}$ & $\mathrm{L} \downarrow$ & $\mathrm{R}$ & A & I \\
\hline Human SREBP-2 & $\mathrm{S}$ & G & $\mathbf{R}$ & $\mathrm{S}$ & $\mathbf{V}$ & $\mathrm{L} \downarrow$ & $\mathrm{S}$ & $\mathrm{F}$ & $\mathrm{E}$ \\
\hline Human SREBP-1a & $\mathrm{P}$ & $\mathrm{G}$ & $\mathbf{R}$ & $\mathrm{N}$ & $\mathbf{V}$ & $\mathrm{L} \downarrow$ & $\mathrm{G}$ & $\mathrm{T}$ & $\mathrm{E}$ \\
\hline Human ATF6 & Q & $\mathrm{R}$ & $\mathbf{R}$ & $\mathrm{H}$ & $\mathbf{L}$ & $\mathrm{L} \downarrow$ & $\mathrm{G}$ & $\mathrm{F}$ & S \\
\hline Arabidopsis pro-S1P Site A & $\mathrm{Y}$ & $\mathrm{Q}$ & $\mathbf{R}$ & $\mathrm{V}$ & $\mathbf{L}$ & $\mathrm{L} \downarrow$ & G & G & S \\
\hline Arabidopsis pro-S1P Site B & $\mathrm{W}$ & $\mathrm{S}$ & $\mathbf{R}$ & $\mathrm{H}$ & $\mathbf{L}$ & $\mathrm{L} \downarrow$ & A & $\mathrm{Q}$ & $\mathrm{K}$ \\
\hline Arabidopsis bZIP17 Site A & $\mathrm{R}$ & $\mathrm{D}$ & $\mathbf{R}$ & $\mathrm{V}$ & $\mathbf{L}$ & $\mathrm{D}$ & $\mathrm{T}$ & $\mathrm{S}$ & $\mathrm{R}$ \\
\hline Arabidopsis bZIP17 Site B & $\mathrm{Q}$ & $\mathrm{N}$ & $\mathbf{R}$ & $\mathrm{R}$ & $\mathbf{I}$ & $\mathrm{L}$ & $\mathrm{R}$ & G & $\mathrm{L}$ \\
\hline Arabidopsis bZIP28 Site A & $\mathrm{K}$ & G & $\mathbf{R}$ & $\mathrm{V}$ & $\mathbf{L}$ & M & $\mathrm{V}$ & G & $\mathrm{D}$ \\
\hline Arabidopsis bZIP28 Site B & $\mathrm{K}$ & $\mathrm{N}$ & $\mathbf{R}$ & $\mathrm{R}$ & $\mathbf{I}$ & $\mathrm{L}$ & $\mathrm{E}$ & $\mathrm{G}$ & $\mathrm{L}$ \\
\hline Arabidopsis bZIP49 Site A & $\mathrm{R}$ & $\mathrm{G}$ & $\mathbf{R}$ & $\mathrm{V}$ & $\mathbf{L}$ & $\mathrm{V}$ & $\mathrm{V}$ & $\mathrm{D}$ & $\mathrm{S}$ \\
\hline Arabidopsis bZIP49 Site B & $\mathrm{K}$ & $\mathrm{N}$ & $\mathbf{R}$ & $\mathrm{R}$ & $\mathbf{I}$ & $\mathrm{L}$ & $\mathrm{S}$ & G & G \\
\hline Arabidopsis RALF23 & $\mathrm{I}$ & $\mathrm{N}$ & $\mathbf{R}$ & $\mathrm{R}$ & $\mathbf{I}$ & $\mathrm{L}$ & $\mathrm{A}$ & $\mathrm{T}$ & $\mathrm{R}$ \\
\hline
\end{tabular}

a) The S1P cleavage sites in human proteins were experimentally demonstrated which are denoted with arrows. The conserved amino acids at P2 and P4 positions are highlighted in bold. S1P, Site-1 Protease; SREBP, Sterol-Regulatory Element Binding Protein; ATF6, Activating Transcription Factor 6; RALF23, Rapid Alkalinization Factor 23.
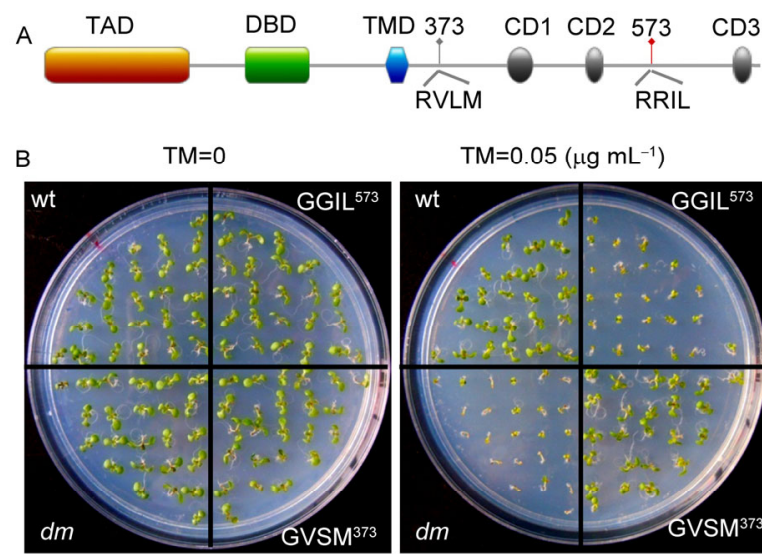

$\mathrm{TM}=0.05(\mu \mathrm{g} \mathrm{mL}-1)$
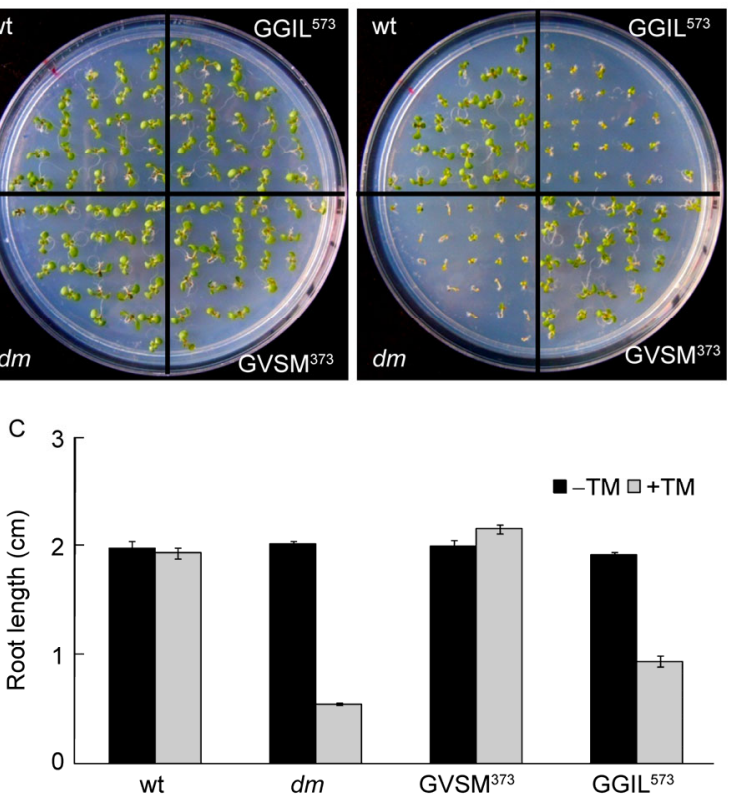

Figure 1 The canonical S1P site of bZIP28 is important for its biological function. A, Diagram of bZIP28 domains. The transcriptional activation domain (TAD), DNA binding domain (DBD), transmembrane domain (TMD) and three sequence conserved domains (CD1-CD3) are illustrated. Two putative S1P cutting sites (RVLM and RRIL) with their respective amino acid positions are also included. B and C, Phenotypic analysis of transgenic plants transformed with mutated forms of bZIP28 in the zip28zip60 $(\mathrm{dm})$ background driven by the bZIP28 native promoter. Plants were photographed (B), roots were pulled out from the growth medium and root length was measured (C) 10 days after germination. Bars depict SE $(n=3)$.
$\mathrm{GVSM}^{373}$ expressing plants grew similarly to the wt (Figure $1 \mathrm{~B}$ and $\mathrm{C}$ ), indicating that mutations at $\mathrm{RVLM}^{373}$ does not affect the function of bZIP28. On the contrary, the GGIL ${ }^{573}$ expressing plants remained as sensitive to TM as the $d m$ plants (Figure $1 \mathrm{~B}$ and $\mathrm{C}$ ), suggesting that the Args at RRIL $^{573}$ site are critical for the function of bZIP28 under ER stress condition. Following $4 \mathrm{~h}$ of TM treatment, the UPR marker genes BiP3 and CNXI were highly upregulated in the wt while not in the $d m$ plants (Figure 2A and B). When the mutated form GVSM $^{373}$ of bZIP28 was expressed in the $d m$ plants, both $B i P 3$ and $C N X 1$ were up-

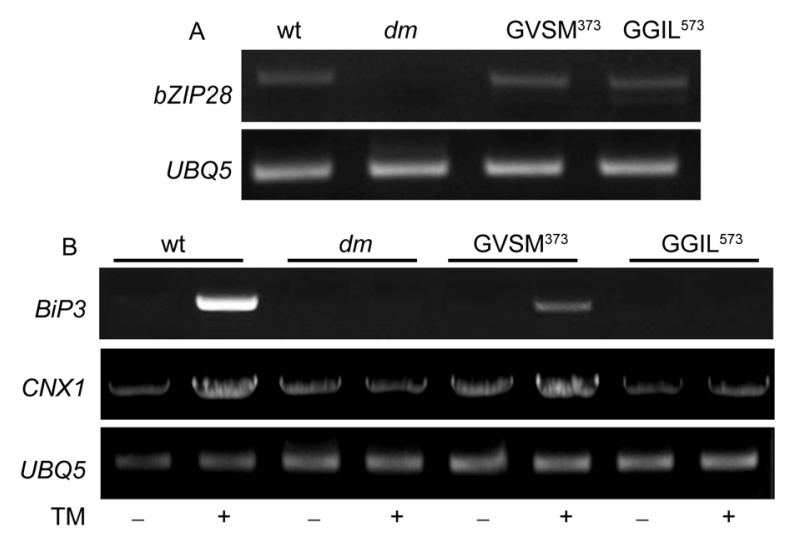

Figure 2 The canonical S1P site of bZIP28 is important for transcriptional regulation of UPR downstream genes. A, Validation of bZIP28 expression in the transgenic plants under normal growth condition. Primer was designed to amplify either the native gene or the transgene. B, Up-regulation of UPR genes by ER stress in the wild-type, zip28zip60 double mutant $(\mathrm{dm})$, and transgenic plants transformed with mutated form of bZIP28 in the $d m$ background. Semi-quantitative RT-PCR was performed and $U B Q 5$ was used as a control. 
regulated by TM treatment (Figure $2 \mathrm{~A}$ and $\mathrm{B}$ ). Partial restoration of UPR downstream gene expression could be explained because bZIP60 was still not functional in the transgenic plants. However, when the mutated bZIP28 form GGIL $^{573}$ was expressed in the $d m$ plants, neither BiP3 nor $C N X 1$ was up-regulated by TM treatment (Figure 2A and B), which is consistent with the ER stress sensitive phenotype of GGIL ${ }^{573}$ expressing plants (Figure $1 \mathrm{~B}$ and $\mathrm{C}$ ). Thus, RRIL $^{573}$ site is crucial for the biological function of bZIP28 during UPR.

\subsection{The S1P cleavage site RRIL ${ }^{573}$ on the lumen-facing domain of bZIP28 is important for its proteolytic pro- cessing}

The bZIP28 precursor is a type II membrane protein with the C-terminus facing the ER lumen. It is processed to a shorter form upon ER stress [22,28]. We were interested in knowing how the RRIL ${ }^{573}$ site contributes to the proteolytic processing. To increase protein stability, green fluorescent protein (GFP) and 4x MYC were fused to the bZIP28 C-terminus (aa 301-675) (M-WT, Figure 3A). Mutated forms of bZIP28 were also generated (Figure 3A). These bZIP28 derivates were over-expressed in Arabidopsis plants driven by the $35 \mathrm{~S}$ promoter. Western blotting analysis indicated that M-WT was processed after DTT treatment (Figure 3B). Although the M-GVSM ${ }^{373}$ precursor was less stable, DTT treatment accelerated the proteolytic processing (Figure 3B). DTT treatment did not induce the processing of

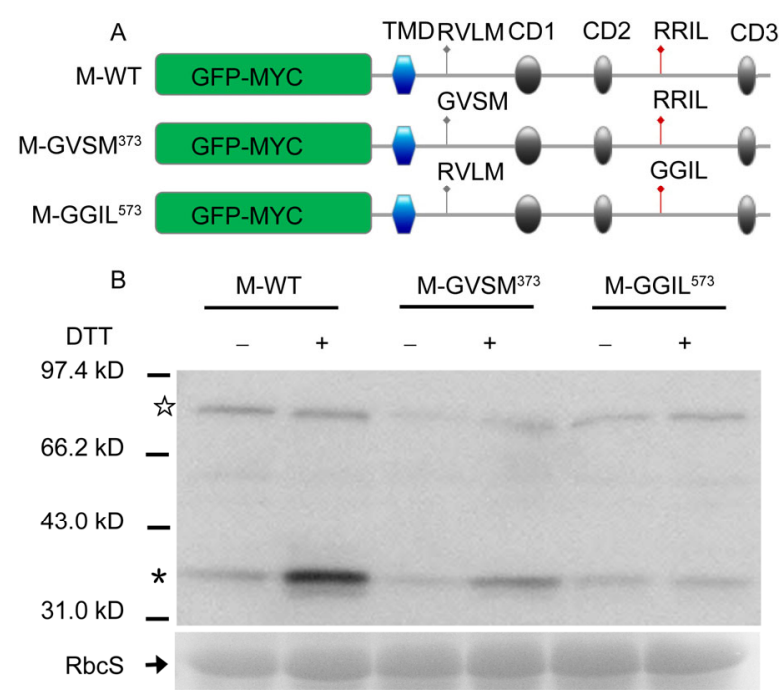

Figure 3 The canonical S1P site of bZIP28 is important for proteolytic processing of bZIP28. A, Diagram showing different forms of bZIP28 derivates. N-terminus of bZIP28 (aa 1-300) is replaced with GFP-MYC in M-WT. Abbreviations of TMD and CD1 to CD3 are the same as in Figure 1. The putative S1P cutting sites and the mutated sites are listed above the illustrations, respectively. B, Western blotting analysis of protein processing of fusion proteins in response to DTT treatment. The star and asterisk denote position of the precursor and the activated form, respectively. Fusion proteins were detected with anti-MYC antibody. Coomassie blue staining of RbcS serves as a loading control.
M-GGIL $^{573}$ (Figure 3B). The consensus motif (R/K)X (hydrophobic) $\mathrm{Z}$ where $\mathrm{Z}$ is any amino acid, preferentially Leu or Thr, but excluding Val, Pro, Glu, Asp, or Cys, was identified for S1P processing in mamalian cells [25,35]. Based on our results, Met at Z site blocks S1P processing in Arabidopsis plant. Previously, the peptide growth factor rapid alkalinization factor 23 (RALF23) was identified as one of the S1P substrates in Arabidopsis, and the two Args at RRIL ${ }^{85}$ site on RALF23 were important for its proteolysis [38]. Our results further demonstrate that the RRIL ${ }^{573}$ site on the lumen-facing domain of bZIP28 is required for its proteolytic processing.

\subsection{The S1P cleavage site RRIL ${ }^{573}$ on the lumen-facing domain of bZIP28 is important for its nuclear relocation}

The hallmark of bZIP28 pathway is the relocation of bZIP28 from the ER to the nucleus via the Golgi [22,28]. The failure of protein processing with M-GGIL ${ }^{573}$ prompted us to investigate the sub-cellular localizations of each bZIP28 derivate. The GFP tagged bZIP28 with native C-terminus (M-WT, Figure 3A) was shown to be translocated from the ER to the nucleus in response to DTT treatment in tobacco (Nicotiana benthamiana) epidermal cells after agro-infiltration (Figure 4). Prior to DTT treatment, M-GVSM ${ }^{373}$ and M-GGIL ${ }^{573}$ were co-localized with the ER marker CD3-959 [30] (Figure 4). Upon DTT treatment, M-GVSM ${ }^{373}$ protein was found in the nucleus (Figure 4), overlaying with a nuclear marker [31]. However, M-GGIL ${ }^{573}$ protein was co-localized with a Golgi body marker CD3-967 [30] after DTT treatment (Figure 4). These results strongly suggest that GGIL ${ }^{573}$ mutation does not affect ER-to-Golgi movement but rather affects proteolytic processing and further nuclear relocation. Previously we have shown that the bZIP28 derivate MLD2 in which the downstream sequences of RRIL ${ }^{573}$ site were deleted, was sufficient for S2P cleavage and ER-to-Golgi-to-nucleus movement [28], indicating that S1P cleavage is to generate a shorter ER lumen-facing C-terminus of bZIP28 for further $\mathrm{S} 2 \mathrm{P}$ processing.

\section{Conclusion}

Regulated nuclear relocation of transcription factors from organelle membrane represents a regulatory control on gene expression in eukaryotic cells. Proteolytic cleavage can be mediated by regulated intramembrane proteolysis. S1P plays important roles in activation of membrane-associated transcription factors, in which pre-cleavage of the lumen-facing sequences by S1P enables the substrate more accessible to further cleavage by S2P [39]. Previously we have demonstrated the important role of S1P in activation of bZIP17 and RALF23 in Arabidopsis [26,38]. In the current paper we have shown that S1P cleavage is also important 


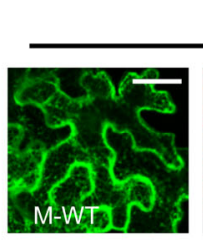

$-\mathrm{DTT}$
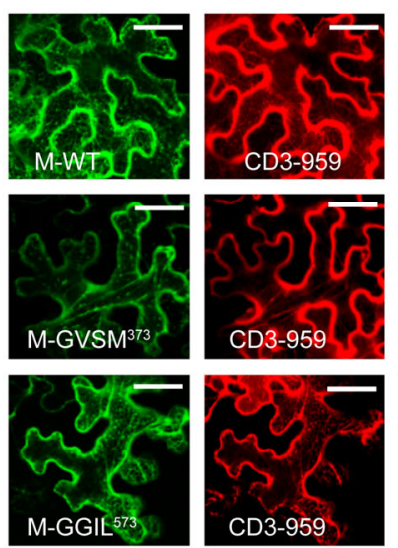
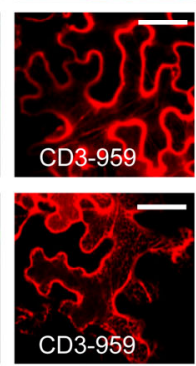
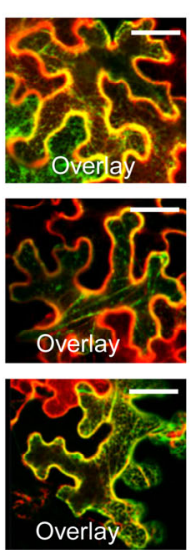
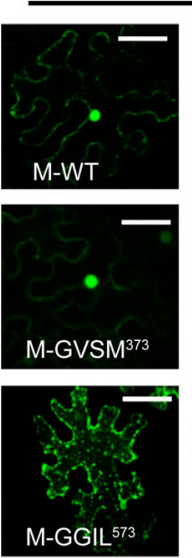

$+\mathrm{DTT}$

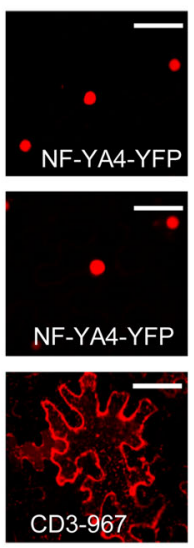

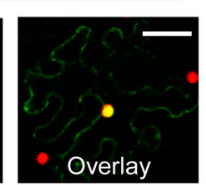
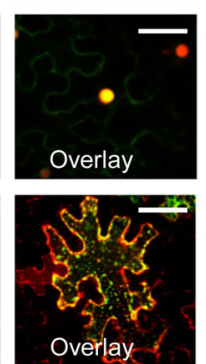

Figure 4 The canonical S1P site of bZIP28 is important for nuclear relocation of bZIP28. Confocal images of various fusion proteins (details in Figure 3A) in tobacco epidermal cells. The ER marker (CD3-959), Golgi marker (CD3-967) and nucleus marker (NF-YA4-YFP) were co-transformed with bZIP28 derivate, respectively. Scale bar, $50 \mu \mathrm{m}$.

for the biological function of the membrane-associated transcription factor bZIP28. The RRIL ${ }^{573}$ site, but not RVLM ${ }^{373}$ site, on the ER lumen-facing domain of bZIP28 is required for the proteolytic processing and nuclear relocation of bZIP28 during plant UPR.

This work was supported by grants from the National Basic Research Program of China (973 Program, 2012CB910500), the National Natural Science Foundation of China (31171157, 31222008), and the Specialized Research Fund for the Doctoral Program of Higher Education (20130071110011).

1 Ron D, Walter P. Signal integration in the endoplasmic reticulum unfolded protein response. Nat Rev Mol Cell Biol, 2007, 8: 519-529

2 Walter P, Ron D. The unfolded protein response: from stress pathway to homeostatic regulation. Science, 2011, 334: 1081-1086

3 Liu JX, Howell SH. Endoplasmic reticulum protein quality control and its relationship to environmental stress responses in plants. Plant Cell, 2010, 22: 2930-2942

4 Hetz C. The unfolded protein response: controlling cell fate decisions under ER stress and beyond. Nat Rev Mol Cell Biol, 2012, 13: 89-102

5 Howell SH. Endoplasmic reticulum stress responses in plants. Annu Rev Plant Biol, 2013, 64: 477-499

6 Vitale A, Boston RS. Endoplasmic reticulum quality control and the unfolded protein response: insights from plants. Traffic, 2008, 9: 1581-1588

7 van Galen P, Kreso A, Mbong N, Kent DG, Fitzmaurice T, Chambers JE, Xie S, Laurenti E, Hermans K, Eppert K, Marciniak SJ, Goodall JC, Green AR, Wouters BG, Wienholds E, Dick JE. The unfolded protein response governs integrity of the haematopoietic stem-cell pool during stress. Nature, 2014, 510: 268-272

8 Cox JS, Shamu CE, Walter P. Transcriptional induction of genes encoding endoplasmic reticulum resident proteins requires a transmembrane protein kinase. Cell, 1993, 73: 1197-1206

9 Mori K, Ma WZ, Gething MJ, Sambrook J. A transmembrane protein with a cdc2+/CDC28-related kinase activity is required for signaling from the ER to the nucleus. Cell, 1993, 74: 743-756

10 Korennykh AV, Egea PF, Korostelev AA, Finer-Moore J, Zhang C, Shokat KM, Stroud RM, Walter P. The unfolded protein response signals through high-order assembly of IRE1. Nature, 2009, 457:
687-693

11 Deng Y, Humbert S, Liu JX, Srivastava R, Rothstein SJ, Howell SH. Heat induces the splicing by IRE1 of a mRNA encoding a transcription factor involved in the unfolded protein response in Arabidopsis. Proc Natl Acad Sci USA, 2011, 108: 7247-7252

12 Nagashima Y, Mishiba KI, Suzuki E, Shimada Y, Iwata Y, Koizumi N. Arabidopsis IRE1 catalyses unconventional splicing of bZIP60 mRNA to produce the active transcription factor. Sci Rep, 2011, 1: 29

13 Moreno AA, Mukhtar MS, Blanco F, Boatwright JL, Moreno I, Jordan MR, Chen Y, Brandizzi F, Dong X, Orellana A, PajerowskaMukhtar KM. IRE1/bZIP60-mediated unfolded protein response plays distinct roles in plant immunity and abiotic stress responses. PLoS One, 2012, 7: e31944

14 Hayashi S, Wakasa Y, Takahashi H, Kawakatsu T, Takaiwa F. Signal transduction by IRE1-mediated splicing of bZIP50 and other stress sensors in the endoplasmic reticulum stress response of rice. Plant $\mathrm{J}$, 2012, 69: 946-956

15 Lu SJ, Yang ZT, Sun L, Sun L, Song ZT, Liu JX. Conservation of IRE1-regulated bZIP74 mRNA unconventional splicing in rice (Oryza sativa L.) involved in ER stress responses. Mol Plant, 2012, 5: 504-514

16 Calfon M, Zeng HQ, Urano F, Till JH, Hubbard SR, Harding HP, Clark SG, Ron D. IRE1 couples endoplasmic reticulum load to secretory capacity by processing the XBP-1 mRNA. Nature, 2002, 415: 92-96

17 Shen XH, Ellis RE, Lee K, Liu CY, Yang K, Solomon A, Yoshida H, Morimoto R, Kurnit DM, Mori K, Kaufman RJ. Complementary signaling pathways regulate the unfolded protein response and are required for $C$. elegans development. Cell, 2001, 107: 893-903

18 Sidrauski C, Walter $\mathrm{P}$. The transmembrane kinase IRE1p is a site-specific endonuclease that initiates mRNA splicing in the unfolded protein response. Cell, 1997, 90: 1031-1039

19 Schroder M, Kaufman RJ. The mammalian unfolded protein response. Annu Rev Biochem, 2005, 74: 739-789

20 Harding HP, Zhang YH, Ron D. Protein translation and folding are coupled by an endoplasmic-reticulum-resident kinase. Nature, 1999, 397: 271-274

21 Ye J, Rawson RB, Komuro R, Chen X, Dave UP, Prywes R, Brown MS, Goldstein JL. ER stress induces cleavage of membrane-bound ATF6 by the same proteases that process SREBPs. Mol Cell, 2000, 6: 1355-1364

22 Liu JX, Srivastava R, Che P, Howell SH. An endoplasmic reticulum stress response in Arabidopsis is mediated by proteolytic processing 
and nuclear relocation of a membrane-associated transcription factor, bZIP28. Plant Cell, 2007, 19: 4111-4119

23 Che P, Bussell JD, Zhou WX, Estavillo GM, Pogson BJ, Smith SM. Signaling from the endoplasmic reticulum activates brassinosteroid signaling and promotes acclimation to stress in arabidopsis. Sci Signal, 2010, 3: ra69

24 Gao HB, Brandizzi F, Benning C, Larkin RM. A membrane-tethered transcription factor defines a branch of the heat stress response in arabidopsis thaliana. Proc Natl Acad Sci USA, 2008, 105: 16398-16403

25 Elagoz A, Benjannet S, Mammarbassi A, Wickham L, Seidah NG. Biosynthesis and cellular trafficking of the convertase SKI-1/S1P: ectodomain shedding requires SKI-1 activity. J Biol Chem, 2002, 277: $11265-11275$

26 Liu JX, Srivastava R, Che P, Howell SH. Salt stress responses in Arabidopsis utilize a signal transduction pathway related to endoplasmic reticulum stress signaling. Plant J, 2007, 51: 897-909

27 Shen JS, Prywes R. Dependence of site-2 protease cleavage of ATF6 on prior site-1 protease digestion is determined by the size of the luminal domain of ATF6. J Biol Chem, 2004, 279: 43046-43051

28 Sun L, Lu SJ, Zhang SS, Zhou SF, Sun L, Liu JX. The lumen-facing domain is important for the biological function and organelle-toorganelle movement of bZIP28 during ER stress in Arabidopsis. Mol Plant, 2013, 6: 1605-1615

29 Clough SJ, Bent AF. Floral dip: a simplified method for agrobacterium-mediated transformation of Arabidopsis thaliana. Plant J, 1998, 16: 735-743

30 Nelson BK, Cai X, Nebenfuehr A. A multicolored set of in vivo organelle markers for co-localization studies in Arabidopsis and other plants. Plant J, 51: 1126-1136
31 Liu JX, Howell SH. bZIP28 and NF-Y transcription factors are activated by ER stress and assemble into a transcriptional complex to regulate stress response genes in Arabidopsis. Plant Cell, 2010, 22: 782-796

32 Sakai J, Rawson RB, Espenshade PJ, Cheng D, Seegmiller AC, Goldstein JL, Brown MS. Molecular identification of the sterolregulated luminal protease that cleaves SREBPs and controls lipid composition of animal cells. Mol Cell, 1998, 2: 505-514

33 Raggo C, Rapin N, Stirling J, Gobeil P, Smith-Windsor E, O'Hare P, Misra V. 2002. Luman, the cellular counterpart of herpes simplex virus VP16, is processed by regulated intramembrane proteolysis. Mol Cell Biol, 2002, 22: 5639-5649

34 Murakami T, Kondo S, Ogata M, Kanemoto S, Saito A, Wanaka A, Imaizumi K. Cleavage of the membrane-bound transcription factor OASIS in response to endoplasmic reticulum stress. J Neurochem, 2006, 96: 1090-1100

35 Toure BB, Munzer JS, Basak A, Benjannet S, Rochemont J, Lazure C, Chretien M, Seidah NG. Biosynthesis and enzymatic characterization of human SKI-1/S1P and the processing of its inhibitory prosegment. J Biol Chem, 2000, 275: 2349-2358

36 Iwata Y, Koizumi N. An arabidopsis transcription factor, AtbZIP60, regulates the endoplasmic reticulum stress response in a manner unique to plants. Proc Natl Acad Sci USA, 2005, 102: 5280-5285

37 Iwata $\mathrm{Y}$, Fedoroff NV, Koizumi N. Arabidopsis bZIP60 is a proteolysis-activated transcription factor involved in the endoplasmic reticulum stress response. Plant Cell, 2008, 20: 3107-3121

38 Srivastava R, Liu JX, Guo HQ, Yin YH, Howell SH. Regulation and processing of a plant peptide hormone, AtRALF23, in Arabidopsis. Plant J, 2009, 59: 930-939

39 Seo PJ, Kim SG, Park CM. Membrane-bound transcription factors in plants. Trends Plant Sci, 2008, 13: 550-556

Open Access This article is distributed under the terms of the Creative Commons Attribution License which permits any use, distribution, and reproduction in any medium, provided the original author(s) and source are credited. 